\title{
A GENERALIZATION OF THE CANONICAL COMMUTATION AND ANTICOMMUTATION RELATIONS
}

\author{
STEVEN ROBBINS
}

\begin{abstract}
The relation $C^{*} C C^{*}-C^{*}=C C^{* 2}$ involving a closed densely defined operator $C$ generalizes both the canonical commutation relation of bosons and the canonical anticommutation relation of fermions. It is shown that the operators satisfying this relation can be classified by an index $p$, $p=0,1,2, \ldots, \infty$.
\end{abstract}

Let $C$ be a closed densely defined operator on a complex Hilbert space. The relations

$$
C^{*} C=C C^{*}+I
$$

and

$$
C^{*} C+C C^{*}=I, \quad C^{2}=0
$$

have been studied in detail. See, for example, [1] and the references given there. Relations (1) and (2) are particularly important because of their physical significance. If $C$ is thought of as a creation operator and $C^{*}$ as the corresponding annihilation operator then relation (1) characterizes bosons while relations (2) characterize fermions. For each of these the selfadjoint operator $n=C C^{*}$ is the "number operator" and at least formally satisfies

$$
\left[n, C^{*}\right]=-C^{*} \text {. }
$$

Since $C$ might be an unbounded operator, and in fact must be if relation (1) is satisfied, equation (3) may only be satisfied on a dense domain. However, for both bosons and fermions the formally equivalent relation

$$
C^{*} C C^{*}-C^{*}=C C^{* 2}
$$

is satisfied if the usual interpretation is made. That is, the domain of the operator on the right is the intersection of the domains of the operators on the left and the equation is satisfied on this set. Relation (4) therefore generalizes both the boson commutation relation (1) and the fermion anticommutation relations (2). It will be proved that the operators satisfying equation (4) can be classified by an index $p, p=0,1,2, \ldots, \infty$. If $p=\infty$ then the boson relation (1) is satisfied. If $p=1$ then the fermion relations (2) are satisfied. The case $p=0$ corresponds trivially to the zero operator.

Received by the editors September 20, 1977 and, in revised form, January 24, 1978. AMS (MOS) subject classifications (1970). Primary 47B25, 47B47; Secondary 81A20.

Key words and phrases. Canonical commutation relations, canonical anticommutation relation. 
If $p$ is a nonnegative integer let $N_{p}=\{0,1,2, \ldots, p\}$ and let $N_{\infty}=\{0,1$, $2, \ldots\}$. Let $p$ be a nonnegative integer or $\infty$ and let $K_{p}$ be a $(p+1)-$ dimensional complex Hilbert space with orthonormal basis $\left\{e_{j}: j \in N_{p}\right\}$. Let $C_{p}$ be the linear operator satisfying

$$
C_{p} e_{j}=\sqrt{j+1} e_{j+1}, \quad j \in N_{p} .
$$

where $e_{p+1}=0$ if $p$ is finite. If $p<\infty$ then $C_{p}$ is well defined. If $p=\infty, C_{p}$ is the closed operator which has the finite linear combinations of the basis elements as a core. For any index $p, C_{p}$ is a closed densely defined operator on $K_{p}$ which can easily be shown to satisfy equation (4).

If $C$ is a closed densely defined operator which satisfies relation (1) or relations (2) then $n=C C^{*}$ and $\bar{n}=C^{*} C$ are commuting selfadjoint operators. The operators $C_{p}$ defined above also have this property. Operators satisfying relation (4) need not a priori have $n$ and $\bar{n}$ commute, although when $C$ is bounded, relation (4) and its adjoint imply that $n$ and $\bar{n}$ commute.

TheOREM. Suppose $C$ is a closed densely defined operator on a complex Hilbert space $K$ which satisfies equation (4) and such that $C C^{*}$ and $C^{*} C$ commute. Then there exist mutually orthogonal closed subspaces $K^{\alpha}$ of $K$ such that $K=\bigoplus K^{\alpha}$,each $K^{\alpha}$ reduces $C$ and for each $\alpha$ there is an index $p$ such that the restriction of $C$ to $K^{\alpha}$ is unitarily equivalent to $C_{p}$.

Proof. Suppose $n=C C^{*}=\int \lambda E(d \lambda)$ is the spectral resolution. Let $\Delta$ be the interval $[0, \beta]$ and suppose $w$ satisfies $E(\Delta) w=w$. It will be shown that for each positive integer $k, C^{*} w \in \operatorname{Dom}\left(n^{k}\right)$ and

$$
(n+1)^{k} C^{*} w=C^{*} n^{k} w .
$$

The proof is by induction on $k$. Since $w \in \operatorname{Dom}\left(n^{2}\right)$ and $n^{2}=C C^{*} C C^{*}$, $w \in \operatorname{Dom}\left(C^{*} C C^{*}\right)$ and $w \in \operatorname{Dom}\left(C^{*}\right)$ so by equation (4) $w \in \operatorname{Dom}\left(C C^{* 2}\right)$. Thus $C^{*} w \in \operatorname{Dom}(n)$ and equation (4) applied to $w$ gives equation (5) with $k=1$. Now suppose equation (5) is satisfied for $k$. $E(\Delta) n^{k} w=n^{k} w$ so $n^{k} w \in \operatorname{Dom}\left(C^{*}\right), C^{*} n^{k} w \in \operatorname{Dom}(n)$ and

$$
\begin{aligned}
C^{*} n\left(n^{k} w\right)-C^{*}\left(n^{k} w\right) & =n C^{*}\left(n^{k} w\right), \\
C^{*} n^{k+1} w-(n+1)^{k} C^{*} w & =n(n+1)^{k} C^{*} w
\end{aligned}
$$

so equation (5) is satisfied for $k+1$.

The following lemma will be used to show that

$$
E((-\infty, \beta-1]) C^{*} w=C^{*} w .
$$

LeMma. Suppose $T$ is a selfadjoint operator on a complex Hilbert space with spectral resolution $T=\int \lambda E(d \lambda)$. Suppose $E([0, \infty)) w=w$. Let

$$
b=\lim _{k \rightarrow \infty}\left\|T^{k} w\right\|^{1 / k} .
$$

Then $b$ is the smallest number such that $E([0, b]) w=w$.

Proof. Suppose the operator $T$ is multiplication by $T(x)$ on a measure 
space with measure $\mu$ and $w=w(x) \in L^{2}(\mu)$. Let $\nu$ be the finite measure defined by $d \nu=|w(x)|^{2} d \mu$ and let $\|\cdot\|_{k}$ represent the $L^{k}(\nu)$ norm. $\left\|T^{k} w\right\|^{1 / k}$ $=\|T(x)\|_{2 k}$. As $k \rightarrow \infty$ this approaches $\|T(x)\|_{\infty}$, the desired value of $b$ and the proof of the lemma is complete.

By equation (5),

$$
\left\|(n+1)^{k} C^{*} w\right\|^{2}=\left\|C^{*} n^{k} w\right\|^{2}=\left\langle C C^{*} n^{k} w, n^{k} w\right\rangle=\left\|n^{k+1 / 2} w\right\|^{2} .
$$

Since $E(\Delta) n^{1 / 2} w=n^{1 / 2} w$, if

$$
b=\lim _{k \rightarrow \infty}\left\|n^{k} n^{1 / 2} w\right\|^{1 / k}
$$

then $b \leqslant \beta$. Thus

$$
\lim _{k \rightarrow \infty}\left\|(n+1)^{k} C^{*} w\right\|^{1 / k} \leqslant \beta,
$$

so $E((-\infty, \beta-1]) C^{*} w=C^{*} w$. Since $E((-\infty, 0))=0$, by starting with any bounded interval $\Delta$ and a nonzero $w$ such that $E(\Delta) w=w$ and applying $C^{*}$ the appropriate number of times, a nonzero vector $v$ is obtained such that $C^{*} v=0$. Thus $n$ has at least one eigenvector with eigenvalue 0 . It will next be shown that every eigenvector of $n$ is in the domain of $\bar{n}$.

Let $\bar{n}=C^{*} C=\int \lambda F(d \lambda)$ be the spectral resolution. Let

$$
\hat{K}=\{x \in K: E(\Delta) x=x \text { for some bounded interval } \Delta\} .
$$

If $n w=\alpha w, w \in \operatorname{Dom}(C)$ and $x \in \hat{K}$ then

$$
\begin{aligned}
\langle C w, n x\rangle & =\left\langle w, C^{*} n x\right\rangle=\left\langle w,(n+1) C^{*} x\right\rangle \\
& =\left\langle(n+1) w, C^{*} x\right\rangle=\left\langle(\alpha+1) w, C^{*} x\right\rangle=\langle(\alpha+1) C w, x\rangle .
\end{aligned}
$$

Since $\hat{K}$ is a core for $n, C w \in \operatorname{Dom}(n)$ and $n C w=(\alpha+1) C w$. Similarly, if $w \in \operatorname{Dom}\left(C^{k}\right)$ then $n C^{k} w=(\alpha+k) C^{k} w$. Now suppose $n u=\alpha u$. Let $M=$ $\{x \in K: \bar{n} x=0\}$. Let $P$ be the projection onto $M$, let $u_{2}=P u$ and $u_{1}=u-$ $u_{2}$. Since $n$ and $\bar{n}$ commute, $P$ commutes with $n$ so $n u_{1}=\alpha u_{1}$ and $n u_{2}=\alpha u_{2}$. It will be shown that $\bar{n} u_{1}=(\alpha+1) u_{1}$.

Let $\beta>0$ and $w=F([0, \beta]) u_{1}$. Since $n w=\alpha w$ and $w \in \operatorname{Dom}(C)$,

$$
(\alpha+1) C w=n C w=C C^{*} C w=C \bar{n} w
$$

so

$$
C(\bar{n} w-(\alpha+1) w)=0 .
$$

Since $w \in M^{\perp}$ and $\bar{n} w \in M^{\perp}, \bar{n} w=(\alpha+1) w$. Since $\beta$ was arbitrary, $\bar{n} u_{1}=$ $(\alpha+1) u_{1}$.

Thus, if $n u=\alpha u$ there exist $u_{1}$ and $u_{2}$ such that $u=u_{1}+u_{2}, n u_{1}=\alpha u_{1}$, $n u_{2}=\alpha u_{2}, u_{1}=(\alpha+1) u_{1}$ and $\bar{n} u_{2}=0$. Since $u \in \operatorname{Dom}(C), n C u=$ $(\alpha+1) C u$ and by induction, for each $k, u \in \operatorname{Dom}\left(C^{k}\right)$ and $n C^{k} u=$ $(\alpha+k) C^{k} u$.

It is now possible to show that $K$ can be written as a direct sum with the desired properties. Let $v$ be a unit eigenvector of $n$ with eigenvalue 0 . Then for each $k, v \in \operatorname{Dom}\left(C^{k}\right), n C^{k} v=k C^{k} v$ and $C^{k} v$ is a sum of two (possibly 
zero) eigenvectors of $\bar{n}$ with eigenvalues 0 and $k+1$. Let

$$
m=\min \left\{k: \bar{n} C^{k} v \neq(k+1) C^{k} v\right\}
$$

or $m=\infty$ if this set is empty. If $m=\infty$ then $C^{*} C^{k} v=k C^{k-1} v$ for all $k$. Let $K^{\prime}$ be the closed subspace of $K$ spanned by the $C^{k} v, k=0,1,2, \ldots$

$$
\left\|C^{k} v\right\|^{2}=\left\langle C^{k} v, C^{k} v\right\rangle=\left\langle C^{*} C^{k} v, C^{k-1} v\right\rangle=k\left\|C^{k-1} v\right\|^{2}
$$

and so $\left\|C^{k} v\right\|^{2}=k$ !. If $e_{k}=(k !)^{-1 / 2} C^{k} v,\left\{e_{k}: k \in N_{\infty}\right\}$ is an orthonormal basis for $K^{\prime}$ and $K^{\prime}$ reduces $C$. The restriction of $C$ to $K^{\prime}$ is unitarily equivalent to $C_{\infty}$.

Next suppose $m<\infty$. Let $w_{m}$ be a unit vector parallel to $P C^{m} v$ so $C w_{m}=0$. Define

$$
w_{k}=C^{*(m-k)} w_{m}, \quad 0 \leqslant k \leqslant m-1 .
$$

Then $n w_{k}=k w_{k}$. Thus

$$
\left\|w_{k}\right\|^{2}=\left\langle C^{*} w_{k+1}, C^{*} w_{k+1}\right\rangle=\left\langle C C^{*} w_{k+1}, w_{k+1}\right\rangle=(k+1)\left\|w_{k+1}\right\|^{2}
$$

and so

$$
\left\|w_{k}\right\|^{2}=(k+1)(k+2) \cdots(m), \quad 0 \leqslant k \leqslant m-1 .
$$

If $K^{\prime}$ is the subspace spanned by the $w_{k}, 0 \leqslant k \leqslant m$, then $K^{\prime}$ reduces $C$ and $K^{\prime}$ has orthonormal basis $\left\{e_{k}: 0 \leqslant k \leqslant m\right\}$ where $e_{k}=w_{k} /\left\|w_{k}\right\|$. The restriction of $C$ to $K^{\prime}$ is unitarily equivalent to $C_{m}$.

Thus there is always a subspace $K^{\prime}$ of $K$ which reduces $C$ and an index $p$ such that the restriction of $C$ to $K^{\prime}$ is unitarily equivalent to $C_{p}$. Since the hypotheses of the theorem are satisfied with $C$ restricted to $K^{\prime \perp}$, an application of Zorn's Lemma gives the desired direct sum.

\section{REFERENCES}

1. C. R. Putnam, Commutation properties of Hilbert space operators and related topics, Ergebnisse der Math. und ihrer Grenzgebiete, Band 36, Springer-Verlag, New York, 1967. MR 36 \#707.

Division of Mathematics, Computer Science, and Systems Design, University of TeXas, SAn ANTONio, Texas 78285 